\title{
ARTICLE
}

\section{Measurement of Neutron Yields from Thick Al and SUS304Targets Bombarded by 5-MeV and 9-MeV Deuterons}

\author{
Keiichi HIRABAYASHI $^{1 *}$, Tomoya NISHIZAWA ${ }^{1}$, Haruhiko UEHARA ${ }^{1}$, Hidetaka HIRANO ${ }^{1}$, Tsuyoshi KAJIMOTO $^{1}$ \\ Nobuhiro SHIGYO ${ }^{1}$, Makoto MAEDA ${ }^{1}$,Takashi YASUMUNE ${ }^{1}$, Keisuke MAEHATA ${ }^{1}$ \\ Yuta TAJIRI ${ }^{2}$, Hiroshi UMISHIO ${ }^{2}$, Shin-ichirou ABE $^{2}$, Yukinobu WATANABE ${ }^{2}$ \\ Kenshi SAGARA ${ }^{3}$, Sunao MAEBARA ${ }^{4}$, Hiroki TAKAHASHI ${ }^{4}$, Hironao SAKAKI $^{4}$ \\ ${ }^{1}$ Department of Applied Quantum Physics and Nuclear Engineering, Kyushu University \\ 744, Motooka, Nishi-ku, Fukuoka 819-0395, Japan \\ ${ }^{2}$ Department of Advanced Energy Engineering Science, Kyushu University, Kasuga, Fukuoka, 816-8580, Japan \\ ${ }^{3}$ Department of Physics, Kyushu University, Hakozaki, Fukuoka, 812-8581, Japan \\ ${ }^{4}$ Japan Atomic Energy Agency, Tokai-mura, Naka-gun, Ibaraki, 319-1195, Japan
}

\begin{abstract}
Double differential thick target neutron yields from 5 and $9 \mathrm{MeV}$ deuteron incidence on aluminum and SUS304st ainless steel were measured at the Kyushu University Tandem Accelerator Laboratory. An aluminum, and a SUS 304 foil which were thick enough for a deuteron to stop in the foils were placed at the center of a vacuum chamber. AnNE213 liquid organic scintillator was employed to detect neutrons emitted from targets. To consider the contribution of scattered neutrons from the floor, we also measured neutron yields with an iron shadow bar located in front of the scintillator. Because incident deuteron beam was not pulsed and the Time-of-Flight method was not applied, the energy spectrum was derived from unfolding the light output spectrum using the FORIST code. The re sponse function of the detector was calculated with the SCINFUL-QMD code. The experimental results were compared with the calculation data of the TALYS and PHITS code, and it turned out that the calculation data does not reproduce the experimental ones satisfactorily.
\end{abstract}

\section{KEYWORDS: deuteron, TTNY, NE213, Unfolding, PHITS, TALYS}

\section{Introduction}

The IFMIF-EVEDA (International Fusion Material Irradiation Facility-Engineering Validation and Engineering Design Activity) ${ }^{1)}$ activities in the framework of EU-Japan Bilateral Agreement for the Broader Approach for Fusion, an accelerator has been developed for demonstration of $9 \mathrm{MeV}$ $125 \mathrm{~mA}$ deuteron beam $^{2}$. Reliable evaluation of radiation dose by the deuteron induced nuclear reactions and analysis of shielding data by deuteron beam are essential for safety license to the accelerator facility. However, experimental data of deuteron induced neutron production yields are scarce below $10 \mathrm{MeV}$.

For niobium, copper, titanium and carbon, $5 \mathrm{MeV}$ and 9 $\mathrm{MeV}$ deuteron induced TTNYs (Thick Target Neutron Yields) were measured in our recent work ${ }^{3)}$. In this paper, deuteron incident TTNYs for aluminum and SUS304 stainless steel target is derived, and compared with the PHITS ${ }^{4)}$ and the TALYS ${ }^{5}$ calculations.

Aluminum is usually used to construct a vacuum duct of the accelerator. SUS304 is also usually used as vacuum chamber of the accelerator. It is important to analyze radiation dose from these target materials bombarded by deuteron beam.

*Corresponding Author, E-mail:shigyo@kune2a.nucl.kyushu-u.ac.jp (C) 2012 Atomic Energy Society of Japan, All Rights Reserved.

\section{EXPERIMENT}

\section{Experimental Setup}

The experiment was performed at the 1 st target room in the Kyushu University Tandem Accelerator Laboratory. The experimental setup is illustrated in Figure 1.

The deuteron beam from the tandem accelerator was delivered to a compact vacuum target chamber in the target room. The chamber was insulated from other experimental apparatus and the ground of the experimental room in order to acquire the deuteron beam current.
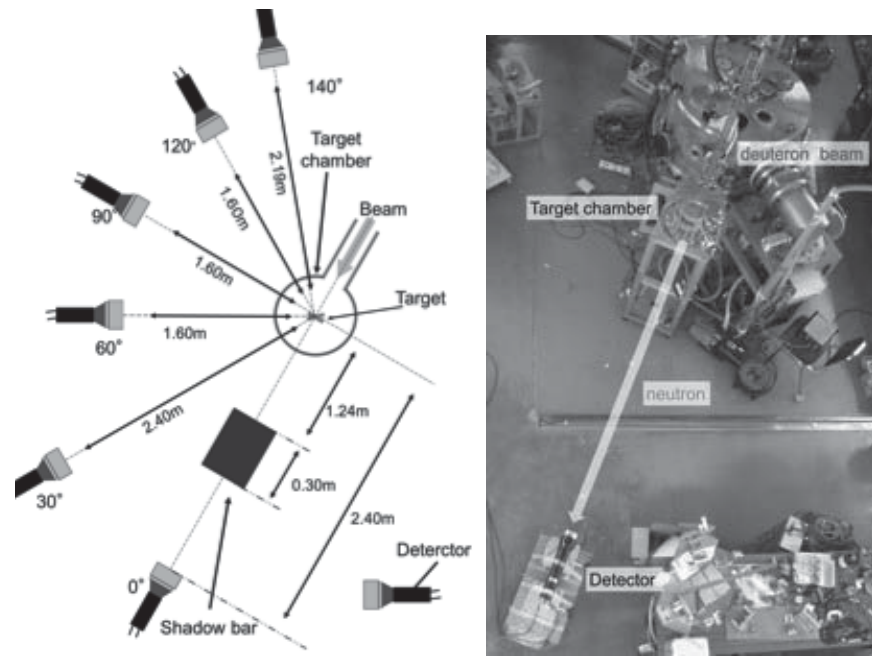

Fig.1 Experimental set up. 
The incident energy of deuteron was $5 \mathrm{MeV}$ for the aluminum and $9 \mathrm{MeV}$ for the SUS304 targets, respectively.

A $0.5 \mathrm{~mm}$ thick aluminum and a $0.2 \mathrm{~mm}$ thickSUS304foils were chosen as targets and placed at the target frame of the vacuum chamber. These target thicknesses were obtained enough to completely stop the incident deuteron by the SRIM code ${ }^{6}$.

An NE213 liquid organic scintillator, $50.4 \mathrm{~mm}$ thick and $50.4 \mathrm{~mm}$ in diameter, optically coupled with a Hamamatsu H1942 or a H6410 photomultiplier was adopted as a neutron detector. The electronic pulse signal as the light output of the scintillator was carried to NIM and CAMAC electronics and recorded as integrated charge information into 2 ADCs with different length of gates to separate neutron and $\mathrm{Y}$ ray events.

The measurement angles were 0,30, 60, 90, 120 and 140 degrees for the aluminum measurement, and 0 and 90 degrees for the SUS304. The neutron detector was placed at each direction. The distances from the target to the neutron detector were varied from 1.6 to $2.4 \mathrm{~m}$ depending on available area of the experimental room.

In order to evaluate contribution of neutrons from floor and wall in the experimental room, the measurement which an iron shadow bar, $150 \mathrm{~mm} \times 150 \mathrm{~mm}$ and $300 \mathrm{~mm}$ thick, was set between the target and the neutron detector for each direction, was also done as background measurement.

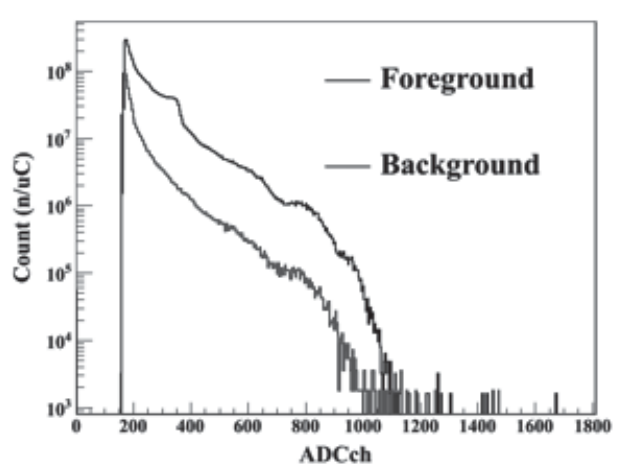

Fig. 2 Examples of raw spectra normalized by the number of incident deuterons for an aluminum target. Upper and lower lines stand for foreground and background measurements, respectively.

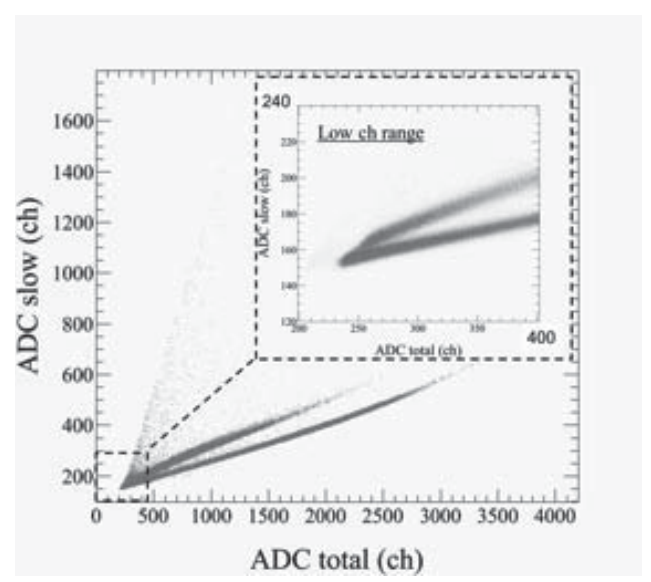

Fig. 3 The two dimensional plot of neutron and $\mathrm{Y}$ ray discrimination using the two gate integration method.
Examples of light output spectra for foreground and shadow bar (background) measurements normalized by the number of incident deuterons for an aluminum target are shown in Figure 2.

\section{Data Analysis}

First, Y ray events were separated in the light output spectra using the two gate integration method because the NE213 scintillator is sensitive to $Y$ rays in addition to neutrons. Figure 3 shows the two dimensional plot of events separated from $Y$ ray ones in low light output region.

Second, the charge spectra of neutron events were converted to the amount in units of electron equivalent, MeVee, using $\mathrm{Y}$ rays from ${ }^{133} \mathrm{Ba}(\mathrm{E} \gamma=0.36 \mathrm{MeV}),{ }^{137} \mathrm{Cs}(\mathrm{E} \gamma=$ $0.66 \mathrm{MeV}),{ }^{60} \mathrm{Co}(\mathrm{EY}=1.17$ and $1.33 \mathrm{MeV})$ and ${ }^{241} \mathrm{Am}-\mathrm{Be}(\mathrm{EY}$ $=4.44 \mathrm{MeV}$ ) standard $\mathrm{Y}$ ray sources. The calibration curve was given by fitting the Compton edge events of these $Y$ rays. The relationship between the charge recorded into an ADC and the light output is shown in Figure 4.

The time-of-flight method was not applied because the deuteron beam was delivered to the target vacuum chamber continuously and it was difficult to produce pulse beam at the accelerator facility. The neutron energy spectra were derived from unfolding the integrated charge spectra as the scintillation light output ones using the response functions of the NE213 scintillator. The response functions were calculated by the SCINFUL-QMDcode ${ }^{7)}$. Figure 5 indicates the calculated response functions.

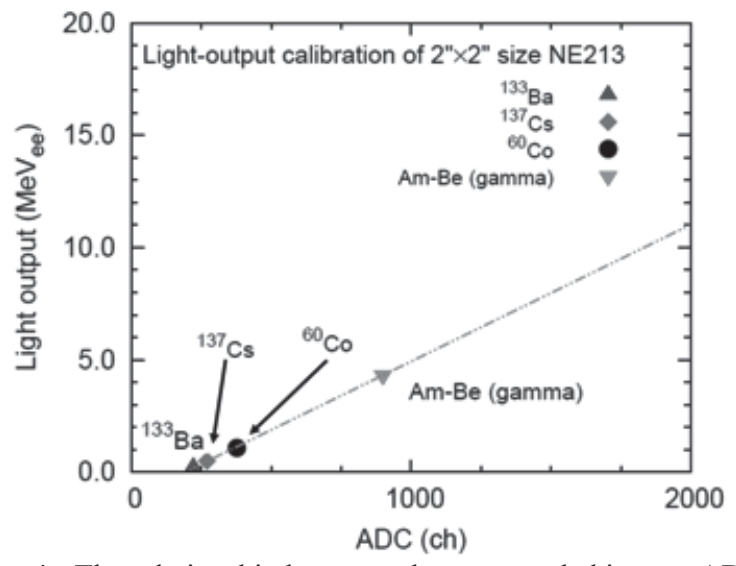

Fig. 4 The relationship between charge recorded into an ADC ch and electron equivalent light output.

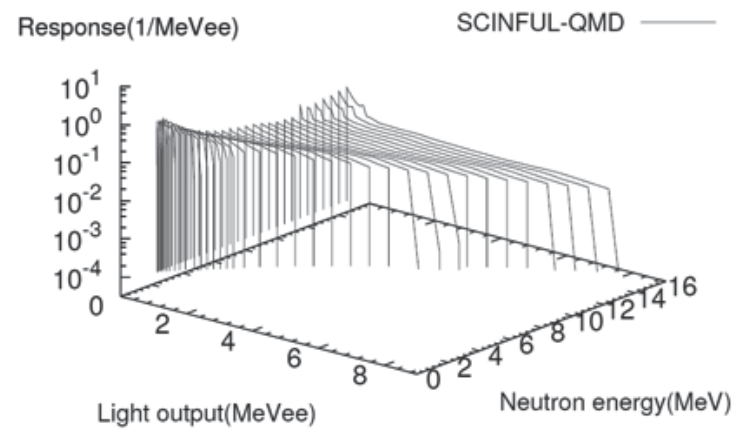

Fig. 5 Example of response function of NE213 using the SCINFUL-QMD code. 
The unfolding of the light output spectra were performed by the FORIST code $^{8}$.

\section{RESULTS AND DISCUSSION}

\section{Validation of Analysis}

For validation of the unfolding method, the measurement of neutrons from an ${ }^{241} \mathrm{Am}-\mathrm{Be}$ and a ${ }^{252} \mathrm{Cfas}$ well known neutron energy spectra was performed.

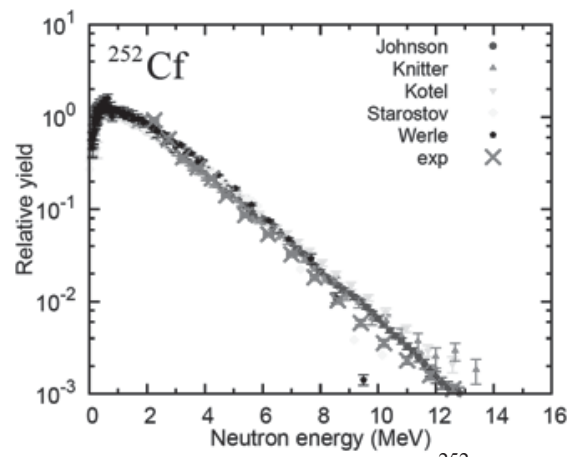

Fig. 6 Measured neutron energy spectraof ${ }^{252} \mathrm{Cf}$ compared with previous experimental data ${ }^{8-12)}$.

Our measured data ${ }^{252} \mathrm{Cf}$ neutron spectrumshows the same tendency as previous data ${ }^{8-12)}$ above $2 \mathrm{MeV}$ in Figure 6. The minimum neutron energy was about 1-2 MeV for both neutron sources.

The experimental energy spectrum of ${ }^{241} \mathrm{Am}-\mathrm{Be}$ neutrons reproduces overall shape of neutron energy spectra by Marxh et al. ${ }^{13)}$ in Figure 7. However,our data doesnot reproduce the structure in the region between 7 and $10 \mathrm{MeV}$. This is because theneutron thick target yield $[\mathrm{n} / \mathrm{MeV} / \mathrm{sr} / \mu \mathrm{C}]$ and the light output bins were roughly divided in this dataanalysis.

\section{TALYS and PHITS calculations}

PHITS and TALYS calculations are performed for the thick target neutron production yield.

In TALYS calculation, the An-Cai potential ${ }^{14)}$ for deuteron incidence was applied. The output of TALYS code is neutron-produced double differential cross-section.

To compare TALYS calculation with our experimental data, equation (1) is used and the energy loss of deuteron in the thick target was considered in this calculation.

$$
\begin{aligned}
\frac{d^{2} Y}{d E_{n} d \Omega_{n}}= & N \int_{0}^{E_{0}}\left[\frac{d^{2} \sigma}{d E_{n} d \Omega_{n}}\right]_{\left(E_{d}, \theta_{n}\right)}\left[\frac{d E_{d}}{d x}\right]^{-1} \\
& \times \exp \left(-\int_{E_{d}}^{E_{0}} \sum_{n o n}\left(E^{\prime}\right) d E^{\prime}\right) d E_{d}
\end{aligned}
$$

where $d^{2} Y / d E_{n} d \Omega_{n}$ is the double differential thick target neutron yields, $N$ the atomic number density of target, $E_{0}$ the incident deuteron energy, $d^{2} \sigma / d E_{n} d \Omega_{n}$ the neutron-produced double differential cross-section, $E_{d}$ the deuteron energy in the target, $\left[d E_{d} / d x\right]$ the stopping power of the target, $\sum_{n o n}\left(E^{\prime}\right)$ the macroscopic total reaction

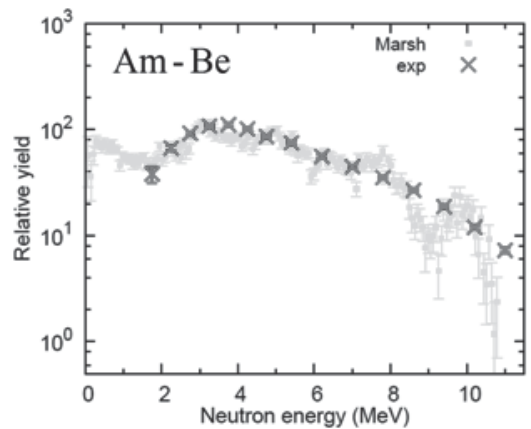

Fig. 7 Measured neutron energy spectra of ${ }^{241} \mathrm{Am}-\mathrm{Be}$ compared with data acquired by Marxh et al. ${ }^{13)}$.

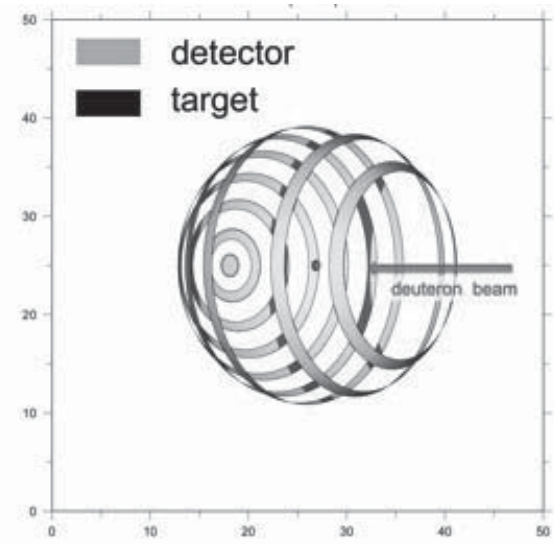

Fig. 8 Calculation geometry in the PHITS calculation.

cross-section.

For PHITS calculation, QMD (Quantum Molecular Dynamics) ${ }^{15)}$ and GEM (Generalized Evaporation Model $)^{16)}$ were adopted. The PHITS calculation geometry is simplified one illustrated in Figure 8. The switching time from QMD to GEM is $150 \mathrm{fm} / \mathrm{c}$.

The NASA's formula ${ }^{17)}$ was applied to total cross-section for nucleus-nucleus collision in the PHITS calculation.

\section{Comparison with the calculated results}

The experimental results of the double differential TTNYs for $5 \mathrm{MeV}$ deuteron incidence on aluminum and9 $\mathrm{MeV}$ incidence on SUS304 are shown in Figure 9 and 10, respectively.

In the case of aluminum target, PHITS calculation agrees with the experimental data except for neutron energies below $7 \mathrm{MeV}$ at $0^{\circ}$. On the other hand, TALYS does not reproduce experimental dataabove7 $\mathrm{MeV}$. Both codes underestimate the experimental values below $7 \mathrm{MeV}$ at $0^{\circ}$. This trend was confirmed for carbon target in our recent work ${ }^{18)}$. This is probably because there is a problem in the calculation of the direct and pre-equilibrium processes for the light nuclei in TALYS.

For SUS304 target, PHITS reproduces energy spectrum approximately. The calculation result by TALYS agrees with the experimental data below $8 \mathrm{MeV}$. However, the code underestimates the experimental data above that energy. With respect to both targets, PHITS and TALYS show different tendency. It is because that both codes do not treat the nature of deuteron stripping reaction accurately. 


\section{Error evaluation}

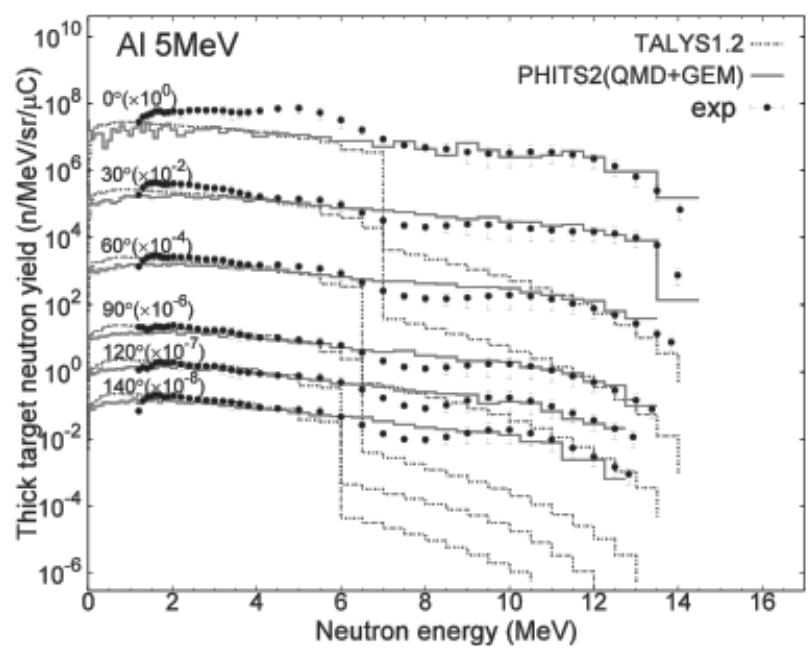

Fig. 9 Double differential neutron thick target yield for $5 \mathrm{MeV}$ deuteron induced on aluminum.

The error bars shown in Figure $\mathbf{9}$ and $\mathbf{1 0}$ include the statistical error and the uncertainty from unfolding by FORIST. The systematic errors were mainly caused by uncertainty of solid-angle, gamma-ray rejection, the accuracy of response function of SCINFUL-QMD, and neutrons scattered by materials and air, the error thus

Table 1Error of Thick Target Neutron Yields.

\begin{tabular}{cc}
\hline Error origin & Error [\%] \\
\hline Solid angle & $2.1-3.2$ \\
\hline Total count of incident deuteron & $<1.0$ \\
\hline Dead time & $<1.0$ \\
\hline Gamma-ray rejection & 5.0 \\
\hline Response function of NE213 & $<17$ \\
\hline Effect of neutron scattered & $<4.0$ \\
\hline Total systematic error & $\mathbf{1 8 . 3 - 1 8 . 5}$ \\
\hline
\end{tabular}

estimated to be $2.1-3.2 \%, 5 \%, 17 \%$ and $4 \%$, respectively. The values are summarized in Table 1.To estimate the effect of neutrons scattered in the air, calculation by PHITS code with the evaluated nuclear data files JENDL-4.0 ${ }^{19)}$ was performed.

\section{SUMMARY}

The double differential TTNYs from $5 \mathrm{MeV}$ deuteron incidence on aluminum and $9 \mathrm{MeV}$ deuteron incidence on SUS304 targets were measured for radiation dose estimation with reasonable validity at the high power deuteron accelerator facility. The light output spectra were unfolded by the FORIST code in order to derive the neutron energy spectra because it was unable to generate pulsed deuteron beam.

PHITS reproduces the experimental data approximately except for energy region below $7 \mathrm{MeV}$ at $0^{\circ}$ for an aluminum target. On the other hand, TALYS underestimates the measured values above $8 \mathrm{MeV}$ for both aluminum and

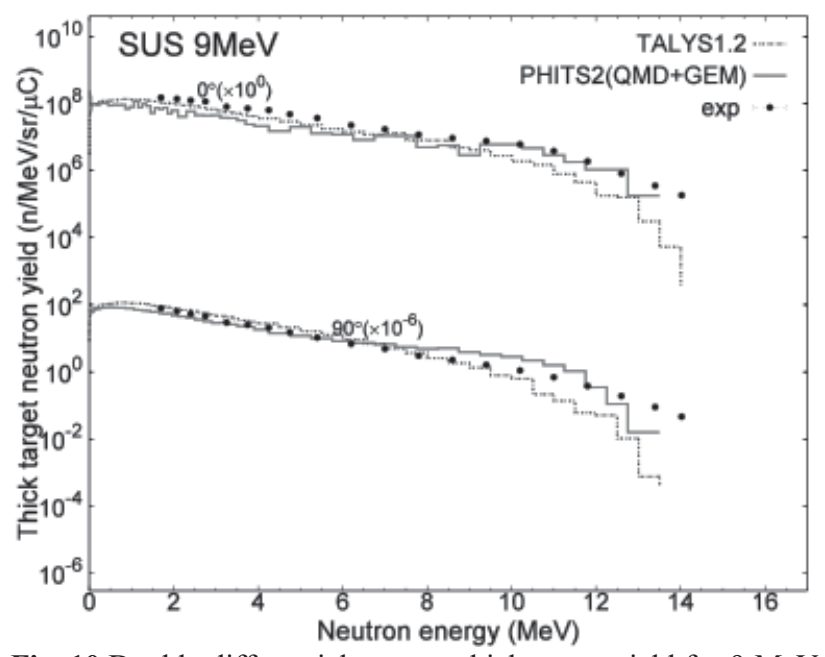

Fig. 10 Double differential neutron thick target yield for $9 \mathrm{MeV}$ deuteron induced onSUS304.

SUS304 targets.

\section{Acknowledgment}

This study was carried out under the joint research between JAEA and Kyushu University for the IFMIF-EVEDA Project.

\section{References}

1) P. Garin, M. Sugimoto and on behalf of the Project Team and all associated Institutes, "Main baseline of IFMIF/EVEDA project," Fusion Eng. Des., 84, 259(2009).

2) A. Mosnier, A. Ibarra and A. Facco, "THE IFMIF-EVEDA ACCELERATOR ACTIVITIES," Proceedings of EPAC08, Genoa, Italy, June 23-27, 2008, THPP077.

3) N. Shigyo, et al., "Measurement of Deuteron Induced Thick Target Neutron Yields at 9 MeV", J. Kor. Phys. Soc., 59, 1725 1728 (2011).

4) K. Niita, et al., "PHITS: Particle and Heavy Ion Transport code System, Version 2.23," JAEA/Data-Code 2010-022 (2010).

5) A.J. Koning, S. Hilaire and M.C. Duijvestijn, "TALYS-1.0," Proc. Int. Conf. Nuclear Data for Science and Technology, April 2007, Nice, France, 211 - 214 (2008).

6) J.F. Ziegler, J.P. Biersack and U. Littmark, "The Stopping and Range of Ions in Solids,"Pergamon Press, New York,2009.

7) D. Satoh, et al., "SCINFUL-QMD: Monte Carlo Based Computer Code to Calculate Response Function and Detection Efficiency of a Liquid Organic Scintillator for Neutron Energies up to $3 \mathrm{GeV}$,'JAEA-Data/Code 2006-023 (2006).

8) R.H. Johnson, et al., "NE-213 Neutron Spectrometry System for Measurements from 1.0 to $20 \mathrm{MeV}$,"Nucl. Instrum. Meth.,145, 337 - 346(1977).

9) H.-H. Knitter et al., "MEASUREMENTS OF THE NEUTRON ENERGY SPECTRUM OF THE SPONTANEOUS FISSION OF ${ }^{252}$ Cf.," Atomkernenergie, 22, 84 - 86 (1973).

10) Kotel'nikova, et al., FEI-575 Obninsk (1975).

11) B.I.Starostov, V.N.Nefedov, A.A.Boytzov, "High precision prompt neutrons spectra measurement for neutrons from Cf-252, U-233, U-235, Pu-239 fission in the energy range 2. - 11. MeV," Proc. 6.All-Union Conf.on Neutron Physics, Kiev, Oct. 1983, 2, 290 (1983).

12) H. Werle and H. Bluhm, "Fission-neutron spectra measurements of ${ }^{235} \mathrm{U},{ }^{239} \mathrm{Pu}$ and ${ }^{252} \mathrm{Cf}$ ', J. Nucl. Energ., 26, 165 - 176 (1972).

13) J.W. Marxh, D.J. Thomas and M. Burke, "High resolution 
measurements of neutron energy spectra from $\mathrm{Am}-\mathrm{Be}$ and Am-B neutron sources,"Nucl. Instrum. Meth., A366, 340 - 348 (1995).

14) D.G. Madland, Proc. a Specialists' Meeting on preequilibrium nuclear reactions, Semmering, Austria, February 1988, 103 (1988).

15) K. Niita, et al., "Analysis of the ( $\mathrm{N}, \mathrm{xN}$ ') reactions by quantum molecular dynamics plus statistical decay model", Phys. Rev., C52, 2620 - 2635 (1995).

16) S. Furihata, "Statistical analysis of light fragment production from medium energy proton-induced reactions", Nucl. Instrum. Meth., B171, 251 - 258 (2000).

17) R.K. Tripathi, F.A. Cucinottac and J.W. Wilson, "Accurate universal parameterization of absorption cross sections III - light systems," Nucl. Insrum. Meth., B155, 349 - 356 (2999), R.K. Tripathi, et al., "Accurate universal parameterization of absorption cross sections II - neutron absorption cross sections," Nucl. Instrum. Meth., B129, 11 - 15 (1997) and R.K. Tripathi, et al., "Accurate universal parameterization of absorption cross sections," Nucl. Instrum. Meth., B117, 347 349 (1996).

18) Y. Tajiri, et al., "Measurement of Neutron Yields from Thick Carbon Bombarded by Deuterons in the MeV Region", Proc. 2011 Fall Meeting of the Atomic Energy Society of Japan, September 2011, Kitakyushu Japan, J51 (2011) [in Japanese].

19) K. Shibata, et al., "JENDL-4.0: A New Library for Nuclear Science and Engineering," J. Nucl. Sci. Technol., 48, 1-30 (2011). 